OPEN ACCESS

Edited by:

Feng Chen,

Augusta University, United States

Reviewed by:

Bo Akerstrom,

Lund University, Sweden

Youjia Yu,

Nanjing Medical University, China

${ }^{*}$ Correspondence:

Abdu I. Alayash

abdu.alayash@fda.hhs.gov

Specialty section:

This article was submitted to

Redox Physiology,

a section of the journal

Frontiers in Physiology

Received: 19 May 2021

Accepted: 27 August 2021

Published: 27 September 2021

Citation:

Alayash Al (2021) The Impact of

COVID-19 Infection on

Oxygen Homeostasis: A

Molecular Perspective.

Front. Physiol. 12:711976.

doi: 10.3389/fphys.2021.711976

\section{The Impact of COVID-19 Infection on Oxygen Homeostasis: A Molecular Perspective}

\author{
Abdu I. Alayash* \\ Division of Blood and Devices (DBCD), United States Food and Drug Administration, Silver Spring, MD, United States
}

The novel coronavirus (2019-nCoV/SARS-CoV-2) causes respiratory symptoms including a substantial pulmonary dysfunction with worsening arterial hypoxemia (low blood oxygenation), eventually leading to acute respiratory distress syndrome (ARDS). The impact of the viral infection on blood oxygenation and other elements of oxygen homeostasis, such as oxygen sensing and respiratory mitochondrial mechanisms, are not well understood. As a step toward understanding these mechanisms in the context of COVID-19, recent experiments revealed contradictory data on the impact of COVID-19 infection on red blood cells (RBCs) oxygenation parameters. However, structural protein damage and membrane lipid remodeling in RBCs from COVID-19 patients that may impact RBC function have been reported. Moreover, COVID-19 infection could potentially disrupt one, if not all, of the other major pathways of homeostasis. Understanding the nature of the crosstalk among normal homeostatic pathways; oxygen carrying, oxygen sensing (i.e., hypoxia inducible factor, HIF) proteins, and the mitochondrial respiratory machinery may provide a target for therapeutic interventions.

Keywords: COVID-19, hemoglobin, oxygen transport, hypoxia inducible factor, mitochondrial function, hypoxia

\section{COVID-19 INFECTION AND OXYGEN HOMEOSTASIS}

The coronavirus, known as Severe Acute Respiratory Syndrome Coronavirus 2 (SARS-CoV-2), is responsible for a multi-systemic disease, called COVID-19. COVID-19 presents with a wide spectrum of clinical signs and symptoms, varying from asymptomatic infection to acute respiratory distress syndrome (ARDS), multifunctional organ dysfunction, and death. Under normal physiological settings the maintenance of cellular levels of oxygen is critical because either insufficient or excess oxygen leads to increased levels of reactive oxygen species (ROS), and therefore, both the delivery and the consumption of oxygen are precisely regulated by many different molecular mechanisms as part of overall oxygen homeostasis. Here, we review the complex and often contradictory literature on the role of blood oxygenation and its impact on other elements of oxygen homeostasis under COVID-19 conditions with a special focus on the delicate balance between oxygen transport/delivery, oxygen sensing (how cells sense oxygen), and finally oxygen consumption (how oxygen is utilized for energy). 
OXYGEN TRANSPORT UNDER NORMAL AND COVID-19 INFECTIOUS CONDITIONS

\section{Oxygen Transport Under Normal Physiology}

Hemoglobin $(\mathrm{Hb})$ is the primary oxygen transporting molecule; some 200-300 million molecules are packed inside circulating red blood cells (RBCs). The binding of oxygen (four oxygen per four hemes) process occurs at the lungs. Binding of the first oxygen (oxyHb; bright red) causes a gradual increase in oxygen-binding affinity until all binding sites on the $\mathrm{Hb}$ molecule are filled in a cooperative manner. As a result, the oxygendissociation curve of $\mathrm{Hb}$ (ODC; also called the oxygen equilibrium curve, OEC) is sigmoidal or S-shaped, as opposed to the normal hyperbolic curve associated with non-cooperative binding (e.g., myoglobin; Dickerson and Gies, 1993; Figure 1).

Carried by arteries, RBCs loaded with oxygen are transported to tissues, where several allosteric modifiers, 2,3-diphosphoglycerate (2,3-DPG), $\mathrm{pH}\left(\mathrm{H}^{+}\right)$, chlorides $\left(\mathrm{Cl}^{-}\right)$, and $\mathrm{CO}_{2}$, collectively force $\mathrm{Hb}$ to unload its oxygen at the respiring tissues. Active metabolism within the respiring tissues generates a considerable amount of $\mathrm{CO}_{2}$ that must be eliminated to avoid acidosis. $\mathrm{Hb}$ returns to the lungs as deoxyHb; it directly carries $\mathrm{CO}_{2}(20 \%)$ back on a single amino acid, Val
1 of the $\beta$ chain of $\mathrm{Hb}$ as a carboamino group. $\mathrm{Hb}$ indirectly helps in the removal of dissolved $\mathrm{CO}_{2}(80 \%)$ as it is being converted from its dissolved acid form and exhaled through the lungs. Carbonic anhydrase, an enzyme found in RBCs catalyzes the reaction between carbonic acid, $\mathrm{CO}_{2}$, and water (Dickerson and Gies, 1993; Alayash, 2004; Balcerek et al., 2020).

Typically, OECs are generated from a plot of the proportion of $\mathrm{Hb}$ in its saturated (oxygen-laden) form on the vertical axis against the prevailing oxygen tension on the horizontal axis (Figure 1). Several important parameters can be derived from these OECs by applying appropriate calculations from the Hill equations. The P50 value when $\mathrm{Hb}$ is half saturated and Hill coefficients (n50) as well as cooperativity (n50) can be derived from these formula. Another important parameter is whether the OECs are left shifted (high oxygen affinity with small P50) or right shifted (low oxygen affinity with a large P50; Alayash, 2004).

\section{Oxygen Transport Under COVID-19 Conditions}

Blood oxygen levels can be assessed by the following oxygenation parameters; $\mathrm{SaO}_{2}, \mathrm{SpO}_{2}$, and $\mathrm{PaO}_{2} . \mathrm{SaO}_{2}$ is the oxygen saturation of arterial blood, while $\mathrm{SpO}_{2}$ is the arterial oxygen pressure as detected by a pulse oximeter. $\mathrm{PaO}_{2}$ represents the arterial blood gas (oxygen tension). The amount of oxygen bound to

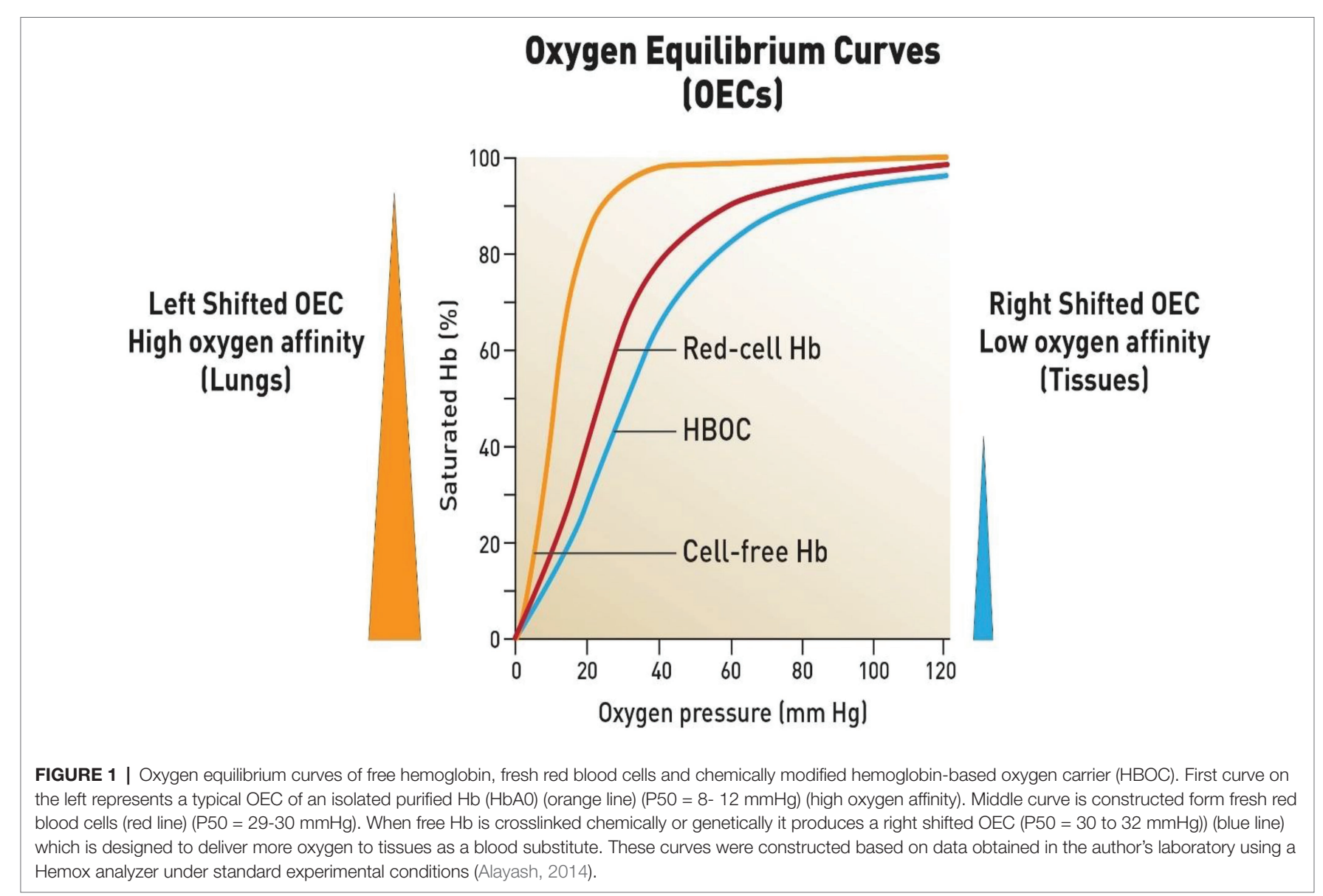


$\mathrm{Hb}$ will increase as the partial pressure of oxygen increases. $\mathrm{SaO}_{2}$, in arterial blood is normally $>95 \%$, whereas at the venous outflow, the venous oxygen saturation $\left(\mathrm{SVO}_{2} ; \mathrm{Hb}\right.$ returning to heart without oxygen) is about 65-75\%. Normal levels of $\mathrm{PaO}_{2}$ and $\mathrm{PaCO}_{2}$ are reported in the range of $75-100$ and $38-42 \mathrm{~mm}$ $\mathrm{Hg}$, respectively (Bickler et al., 2017).

In a recent study on several COVID-19 patients (three patients) ranging in age from 58 to 74 years, the following oxygenation parameters were reported; $\mathrm{SaO}_{2}$ ranged between 69 and $75 \%, \mathrm{SVO}_{2}$ ranged between 68 and 76, and $\mathrm{SPO}_{2}$ it was reported to be between 68 and $76 \%$. Partial pressure of oxygen and $\mathrm{CO}_{2}$ were reported to be between 36 and 45 and $34-40 \mathrm{~mm} \mathrm{Hb}$, respectively (Tobin et al., 2020).

Accordingly, novel treatments have been considered in order to improve Hb-based delivery of oxygen molecules to peripheral tissues in COVID-19 patients. This includes hyperbaric oxygen therapy that significantly increases oxygen levels in the blood independently of $\mathrm{Hb}$ levels and improves tissue oxygenation (Pan et al., 2020). Packed RBCs for transfusion or injected erythropoiesis-stimulating agents that can significantly raise blood $\mathrm{Hb}$ levels by increasing the numbers of RBCs and improving tissue oxygenation have been suggested (Geier and Geier, 2020).

Manufacturers of $\mathrm{Hb}$-based blood substitutes have also promoted the use of oxygen therapeutics during the current COVID-19 pandemic (Weiskopf et al., 2020; Lupon et al., 2021). These oxygen therapeutics have a long shelf-life and can be stored at room temperatures for long durations.

\section{Contested Effects of Severe COVID-19 Infection on Oxygen Transport and Red Blood Cell Integrity}

The question that remains unanswered is whether we know the full impact of COVID-19 infection on the blood oxygenation parameters in the first place. One of the early studies (Daniel et al., 2020) on $\mathrm{Hb}$ oxygen affinity measurements were recorded in 14 patients with COVID-19 and reported similar blood oxygenation to the values obtained from 11 control participants. The oxygen affinity was measured in vitro using the automated Hemox Analyzer under standard conditions [pH (7.4) and room temperature]. The (P50) values were obtained directly from the analyzer, without adjustments for physiological changes in $\mathrm{CO}_{2}$ or $\mathrm{pH}$ in vivo, which could be important in COVID-19.

Clinical data, specifically blood oxygen parameters from 21 critically ill COVID-19 patients and 21 non-COVID-19 ARDS (patient controls), were used to generate Hb-ODCs from direct measurements of venous blood gases. The ODC curve generated from the COVID-19 cohort matched the normal sigmoidal ODC well from normal subjects. This comparison of ODC is not altered in patients with COVID-19 admitted to the ICU. Corresponding lab values of $\mathrm{Hb}$, total bilirubin, ferritin, iron, and $\mathrm{LDH}$ were similar between ICU patients with COVID-19 and those with ARDS without COVID-19 (DeMartino et al., 2020).

To assess alterations in the in vivo $\mathrm{Hb}$ oxygen affinity, a retrospective, observational analysis of all arterial and venous blood gases obtained from all intubated and ventilated patients $(n=43)$ with severe COVID-19 in an intensive care unit was also reported (Vogel et al., 2020). In this study, the P50 values were calculated using the Hill equation after correcting for $\mathrm{pH}$, temperature, and base excess derived from a Roche Blood gas analyzer and compared to the normal value (for $\mathrm{pH} 7.4$, $37.0^{\circ} \mathrm{C}$ and $\mathrm{pCO}_{2} 40 \mathrm{mmHg}$ ). Using a reported normal $\mathrm{P} 50$ of $26.7 \mathrm{~mm} \mathrm{Hg}$, distribution of P50 values calculated using Hill equation from measured $\mathrm{SpO}_{2}$ and $\mathrm{SaO}_{2}$ showed a left shift in OECs (P50 values ranged between 14.7 and $25.7 \mathrm{mmHg}$ ) for the bulk of the patients and smaller number of patients exhibited a right shift of oxy $\mathrm{Hb}$ affinity from the standard P50 value (Vogel et al., 2020).

In a more recent retrospective observational study, blood samples from 100 subjects (COVID and non-COVID) from each group were analyzed. The time-course of P50 between days 1 and 18, showed no significant differences among the groups. Median P50 at baseline was $26 \mathrm{mmHg}(25.2-26.8)$ vs. $25.9 \mathrm{mmHg}$ (24-27.3), respectively (Gille et al., 2021).

Another unresolved aspect of this disease is the impact COVID-19 infection on the health and integrity of circulating RBCs in these patients. Early modeling studies by Liu and Li (2020), predicted the spike S1 protein can interact with $\mathrm{Hb}$ to reduce both oxygen affinity and total $\mathrm{Hb}$ content, but the work was subsequently questioned due to a lack of experimental support (Read, 2020).

Using state-of-the-art metabolomics, proteomics, and lipidomics approaches (Thomas et al., 2020), the impact of COVID-19 was investigated from RBCs obtained from 23 healthy subjects and 29 COVID-19 patients. RBCs from COVID-19 patients had increased levels of glycolytic intermediates, accompanied by oxidation and fragmentation of ankyrin, spectrin beta, and the N-terminal cytosolic domain of band 3 (AE1). These increases in RBC glycolytic proteins were reported to be consistent with a theoretically improved capacity of $\mathrm{Hb}$ to off-load oxygen modulation by high-energy phosphate compounds, perhaps to counteract COVID-19-induced hypoxia. It is also known that the $\mathrm{N}$ - terminus of AE1 stabilizes deoxyHb and finely tunes oxygen off-loading and metabolic rewiring toward the hexose monophosphate shunt, in RBCs from COVID-19 patients (Thomas et al., 2020).

The basic understanding of cellular oxygen availability under COVID-19 conditions is not fully understood and is rather controversial. For oxygen to reach cells and ultimately the mitochondria for oxidative metabolism it requires normal blood oxygen carrying capacity as determined by $\mathrm{Hb}$ level, and its ability to release oxygen to the tissue (Kulow and Fähling, 2021). It appears as indicated, by only a few investigations so far, that both oxygen carrying capacity as well RBC integrity, are affected under COVID-19 conditions.

\section{OXYGEN SENSING MECHANISMS AND THE HYPOXIA INDUCIBLE FACTOR-1 $\alpha$}

Hypoxia inducible factor (HIF) together with its iron containing enzyme, prolyl hydroxylase (PHD) has been identified as key 
elements in tissue sensing mechanisms. Under normal oxygen tension (normoxia), the transcriptional activity of HIF- $1 \alpha$ is halted by a process of hydroxylation (at Pro564 and/or Pro402), which involves the non-heme iron $\alpha$-ketoglutarate dependent dioxygenases, PHD (Semenza, 2007). The iron center $\left(\mathrm{Fe}^{2+}\right)$ of the PHD enzyme undergoes redox transition to ferric $\left(\mathrm{Fe}^{3+}\right)$ and ferryl $\left(\mathrm{Fe}^{4+}\right)$, which appears to be the main regulator of HIF- $1 \alpha$ protein stability and control (Chowdhury et al., 2009). This process is followed by ubiquitination of some lysine residues prior to the final degradation by proteasome. During the initial stages of hypoxia, HIF, which contains the two subunits, an $\alpha$-subunit that quickly degrades in the presence of oxygen (its half-life is less than $5 \mathrm{~min}$ in $21 \%$ oxygen) and a more stable $\beta$ is translocated to the nucleus, where it binds to hypoxia response element a process that results in activation of a number of target genes, which collectively function to correct oxygen deficits. This specifically includes a group of genes participating in glucose metabolism, control of intracellular $\mathrm{pH}$, angiogenesis, erythropoiesis, and mitogenesis (Semenza, 2007; Figure 2).

One of the early experiments demonstrating a link between blood oxygen levels and HIF involved exchanging $50 \%$ of rat blood with non-oxygen-carrying pentastarch. In this model, hypoxia led to increased expression of HIF-1 $\alpha$, and several other target genes such as endothelial nitric oxide synthase (eNOS) and vascular endothelial growth factor (VEGF) in the cerebral cortex of these mildly anemic rats (McLaren et al., 2007). To test whether an oxygen carrier cross talks with HIF under hypoxic conditions in an in vivo setting, a class of therapeutics designed to correct oxygen deficit under conditions of anemia and traumatic blood loss were used. A correlation between the oxygenation/oxidation states of the cell-free $\mathrm{Hb}$ and renal HIF- $1 \alpha$ binding activity in a rat and guinea- pig model of $50 \%$ blood replacement was observed (Buehler et al., 2007). Using a more severe model of hemodilution designed specifically to induce hypoxia ( $80 \%$ ET; exchange transfusion), a polymerized oxygen carrying $\mathrm{Hb}$ suppressed HIF expression in kidney tissues for approximately $5 \mathrm{~h}$ post infusion as compared to non-oxygen carrying hetastraches. There was a corresponding depression in EPO gene expression as well EPO serum levels in these animals followed by gradual rebound in EPO as $\mathrm{Hb}$ is cleared (half time of $\sim 16 \mathrm{~h}$ ) and/or due to complete oxidation of the protein (Manalo et al., 2008). These studies collectively demonstrated in an in vivo setting the delicate balance that existed between oxygen content of blood and oxygen sensing mechanisms in tissues.

\section{HIF and Oxygen Sensing Pathways Under COVID-19 Infection Conditions}

Globally, hypoxia impacts organs at the cellular and subcellular levels. Hypoxic responses and hypoxia-mediated elements have been reported in severe cases of COVID-19, which may progress to ARDS, ultimately leading to end organ dysfunction and failure (Serebrovska et al., 2020). At the cellular level, once cells are infected with SARS-CoVID, accumulation of HIF- $1 \alpha$ may occur due to increased expression as well as inhibited proteasome degradation (Vassilaki and Frakolaki, 2017). In addition, a possible secondary bacterial infection during the later phase of COVID-19 may result in the stabilization of
HIF- $1 \alpha$ in macrophages via the activation of toll-like receptor 4 (TLR4) and decrease in prolyl hydroxylase mRNA in severe inflammatory vascular disease (Peyssonnaux et al., 2007). Subsequent local hypoxia events may also occur when leukocytes are activated in response to secreted interferon as well as to accumulation of pathogen associated molecular patterns (PAMPs) and damage associated molecular patterns (DAMPs) as part of the normal innate immune responses.

The entry of the SARS-CoV-2 virus inside cells is facilitated by angiotensin converting enzymes (ACE) found in the lungs, kidneys, heart, and arteries (Gheblawi et al., 2020). Under hypoxic conditions ACE-1, is upregulated by the (HIF-1); meanwhile, the expression of ACE-2 is markedly decreased (Figure 2). It has been suggested that increased levels of ACE-2 were positively associated with COVID-19 infection (Koch et al., 2020).

However, there appears to be a disconnect according to recent reports between hypoxemia (low arterial oxygen saturation) in those patients who generally experience only mild respiratory distress or difficulty in breathing (dyspnea; Tobin et al., 2020). Several other seemingly contradictory observations have also been made. For example, severe hypoxemia in COVID-19 patients does not necessarily cause tissue hypoxia. This may be due to a lack of understanding in the pathophysiology of what has been described as "happy hypoxia" (Rahman et al., 2021). However, a variety of factors have been reported to play a possible role in this phenomenon that includes, a left shift in Hb's OECs, low levels of $\mathrm{CO}_{2}$, decreased $\mathrm{pH}$, and potentially changes in 2,3-DPG levels (Dhont et al., 2020).

\section{MITOCHONDRIAL RESPIRATION UNDER NORMAL AND COVID-19 INFECTION CONDITIONS}

Mitochondria are essential cellular organelles that play important roles in regulating cellular energy, metabolism, survival, and proliferation (Nakhle et al., 2020). Their main energy currency is adenosine triphosphate (ATP) generated by the electron transport chain (ETC). ETC is the metabolic bottle neck, where breakdown metabolites resulting from food degradation pathways (i.e., glycolysis, amino acid, and lipid degradation) followed by the tricarboxylic acid cycle (TCA) are processed. These metabolites are then transformed into protons (electrons) tunneled through the ETC complexes (I, II, III, and IV) that are largely bound to the inner membrane of the mitochondria. As electrons shuttled through the ETC chain, ATP is produced as a result of a wellorchestrated oxidation phosphorylation (OXPHOS) process. Mitochondria also provide intracellular signaling messages through ROS production (Martínez-Reyes and Chandel, 2020). As expected with such a central cellular role, mitochondrial dysfunctions have been linked to many different diseases. Quantifying bioenergetic health index (BHI) has become an important tool in patient populations as laboratory methods have been developed to begin an integrated approach in cells isolated from human blood to establish a quantitative assay of mitochondrial function 


\section{Oxygen homeostasis during COVID-19}

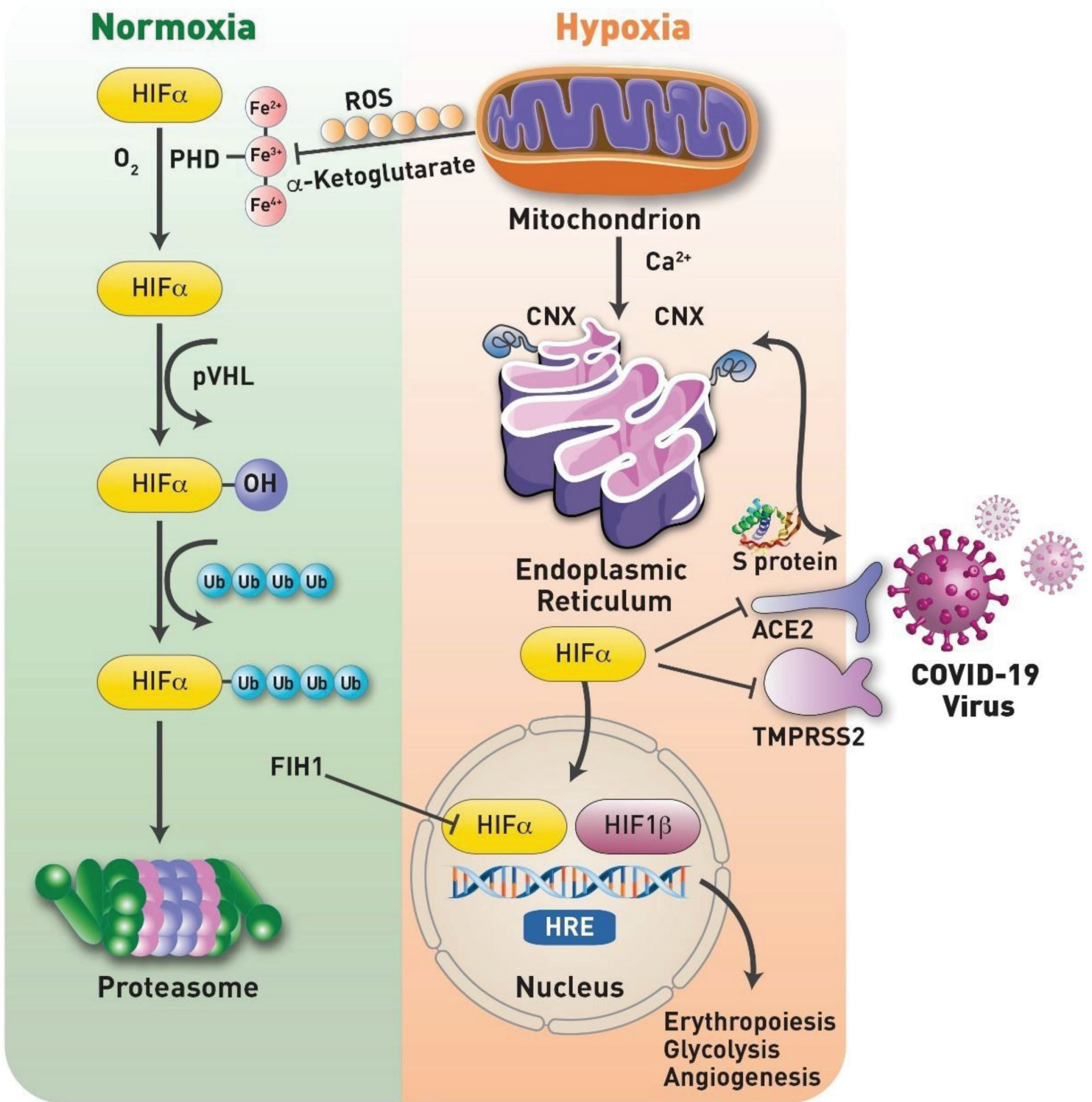

FIGURE 2 | Oxygen homeostasis during COVID-19. Under normal physiological oxygen levels (normoxia), the first step in the hypoxia-inducible factor (HIF-1 $\alpha$ ) degradation is an oxygen-dependent interaction with the von Hippel- Lindau (VHL) tumor suppressor protein (pVHL) complex. This requires hydroxylation of two HIF- $1 \alpha$ proline residues by a family of $\alpha$-ketoglutarate-dependent dioxygenases, prolyl hydroxylases (PHDs), which requires oxygen, iron, ascorbate and $\alpha$-ketoglutarate to function. The catalytic cycle in the PHD involves the transition of its iron from $\mathrm{Fe}^{2+}$ to $\mathrm{Fe}^{3+}$ and most active $\mathrm{Fe}^{4+}$ states. Following hydroxylation, $\mathrm{HIF}-1 \alpha$ subunits are polyubiquitylated by $\mathrm{pVHL}$ and targeted for proteasomal degradation. Under conditions of oxygen deprivation, mitochondria increase their production of reactive oxygen species (ROS). Serving as signaling molecules, these ROS inhibit the hydroxylation of HIF-1 $\alpha$ and, thus, preventing its proteasomal degradation. The ROS are known to target the iron transition required for the protein hydroxylation and/or specific cysteine residues for the irreversible oxidation of this amino acid that results in the deactivation of the protein. Stabilized HIF-1 $\alpha$ is able to translocate to the nucleus where it forms a heterodimeric complex with HIF-1 $\beta$ /ARNT. Factor inhibiting HIF-1 (FH-1), is a novel protein that interacts with $\mathrm{HIF}-1 \alpha$ and mediates repression of $\mathrm{HIF}-1 \alpha$ transcriptional activity. The transcription factor complex activates the expression of several genes, such as Epo and Vegf, to increase oxygen delivery and many others including pyruvate dehydrogenase 1 (PDK1) to reduce oxygen consumption. These factors secure oxygenation to the tissues. At the onset of infection and activation of HIF-1 $\alpha$ which is shown to suppress the angiotensin-converting enzyme 2 (ACE2) receptor and transmembrane protease serine 2 (TMPRSS2) and upregulate disintegrin and metalloproteinase domain-containing protein 17 (ADAM17). In addition, the protein targets of HIF-1 $\alpha$ are involved with the activation of pro-inflammatory cytokine expression and the subsequent inflammatory process. In addition, coronaviruses, as well as other viruses, use the host's chaperone system for the folding of their proteins and for viral assembly, translocation to the nucleus, and other vital steps. Calnexin (CNX) is one of those chaperone proteins of the endoplasmic reticulum (ER) system that ensures the proper folding of viral glycoproteins. In addition, $\mathrm{CNX}$ is involved in trafficking of calcium $\left(\mathrm{Ca}^{2+}\right)$ during ER- mitochondria $\mathrm{Ca}^{2+}$ flux, maintaining the necessary redox balance within the mitochondria. 
that will have the power to predict disease progression and response to treatment (Chacko et al., 2014).

Mitochondrial dysfunction and subsequent pathogenesis due to multi-organ failure has been linked to COVID-19 infection and is under active investigation. Recent reports suggest that the SARS-CoV-2 virus proteins such as the spike protein interacts with host cell mitochondrial proteins leading to loss of membrane integrity and dysfunction in the bioenergetics of the mitochondria. These mitochondrial proteins may also serve as damage-associated molecular pattern (DAMP) molecules, which activate innate immunity (Shenoy, 2020).

A recent paper suggested that COVD infection evade host immunity by "hijacking" mitochondrial pathways. Specifically, this effect is manifested into critical areas, such as the lungs where mitochondrial dysfunction due to SARS-CoV2 infection possibly contributes to pulmonary tissue damage, deterioration of pulmonary function, and airway hypoxia. Impaired mitochondria in the carotid bodies may also worsen hypoxemia due to impaired oxygen sensing and result in a compromised chemoreflex (Burtscher et al., 2020). In a recent study, functional mitochondrial changes in live peripheral blood mononuclear cells (PBMCs) from patients with COVID-19 were investigated. The investigators demonstrated mitochondrial dysfunction, metabolic alterations with an increase in glycolysis, and high levels of mitokine in PBMCs from patients with COVID-19. These data suggest that patients with COVID-19 have a compromised mitochondrial function and an energy deficit that is compensated by a metabolic switch to glycolysis. This metabolic manipulation by SARS-CoV-2 triggers an enhanced inflammatory response that contributes to the severity of COVID-19 symptoms (Ajaz et al., 2021).

Using a conventional Seahorse XF analyzer, two important parameters were measured in the presence of specific mitochondrial activators and inhibitors; oxygen consumption rate (OCR), a measurement of mitochondrial respiration, and extracellular acidification rate (ECAR), which correlates to the number of protons released from the cell (due to contribution from glycolysis and the Krebs cycle; Ajaz et al., 2021). The authors reported a lack of significant differences in basal and stressed OCR among COVID-19-positive individuals' patients. Stressed ECAR was also higher as compared with controls. This high basal and stress ECAR suggest that the PBMCs of patients with COVID-19 depend on glycolysis for energy (Ajaz et al., 2021).

\section{COVID-19 INFECTION DISRUPTS HOMEOSTATIC PATHWAYS-A WORKING MODEL}

Figure 1 represents a working model that combines the major pathways of homeostasis under normal oxygen tension (normoxia) and during oxygen deprivation (hypoxia) and the impact of COVID-19 infection on these pathways. Among the key signaling molecules that bridge the mitochondrial and oxygen sensing pathways are the mitochondrial ROS. ROS are generated as a function of decreasing oxygen tension inhibit HIF-1a prolyl hydroxylation and degradation.
Therefore, mitochondrial electron transport and subsequent oxidative phosphorylation are interconnected as signaling mechanisms conveying cellular oxygen availability to oxygen sensing pathways (Maltepe and Saugstad, 2009). Increases in other glycolytic intermediates (e.g., $\alpha$-ketoglutarate) levels results in HIF stabilization (Lee et al., 2020).

Another key player activated during hypoxia is calnexin (CNX); this membrane-bound endoplasmic reticulum (ER) chaperone protein, ensures the proper folding of glycoproteins destined for the plasma membrane or secretion (Kozlov and Gehring, 2020). The SARS-CoV spike glycoprotein (S protein), a key molecule for viral entry, binds to CNX, conferring infectivity on SARS-CoV (Fukushi et al., 2012). Under cellular stress induced by hypoxia, cells display enhanced and sustained expression of the ER stress-related chaperone proteins $\mathrm{CNX}$ (Figure 1). CNX controls mitochondrial positioning and respiration, and it appears to be yet another molecule that can bridge the gap between oxygen sensing and mitochondrial pathways even closer (Gutiérrez et al., 2020).

Toward this end, we have recently begun to investigate the interplay between oxygen transport, oxygen sensing, and mitochondrial metabolism in human pulmonary arterial endothelial cells (HPAEC) by exploring, first the impact of COVID-19 spike protein on these parameters in the presence of $\mathrm{Hb}$ and under normoxic and hypoxic cell culture conditions. We found that the spike protein alone induced changes in cells without the entry of the virus and that cell-free $\mathrm{Hb}$ did not attenuate the effects of the SARS-CoV-2 S1 spike protein in vitro (Jana et al., 2021). We also begun to investigate the three elements of homeostasis (oxygen transport, oxygen sensing, and mitochondrial pathways) in lungs from hamster model of COVID-19 infections (Selvaraj et al., 2021) to verify the interconnectivity of these pathways in living biological systems.

COVID-19 infection clearly impacts oxygen sensing elements as seen in changes with HIF-target genes as well as mitochondrial respiration in response to low oxygen levels in circulation. Once these pathways and their relationships are established in clinical settings therapeutically targeting each individual pathway or as a group would be possible. Manipulation of RBC's oxygen affinity in circulation can be targeted by a number of allosteric modifiers that can shift OECs to either to the left or to the right of normal OECs (Woyke et al., 2021). Stabilizing HIF under normoxic conditions are also feasible by targeting the heme iron of the enzyme PHD or some of the small molecule drugs that are currently used in cancer therapy (Bao et al., 2021).

Finally, mitochondrial or glycolytic intervention are also feasible by targeting a site or sites in the mitochondrial respiratory chain using small molecule drugs (Andreux, 2013).

\section{DATA AVAILABILITY STATEMENT}

The original contributions presented in the study are included in the article/supplementary material, further inquiries can be directed to the corresponding author. 


\section{AUTHOR'S NOTE}

This article reflects the views of the author and should not be construed to represent FDAs views or policies.

\section{REFERENCES}

Ajaz, S., McPhail, M. J., Singh, K. K., Mujib, S., Trovato, F. M., Napoli, S., et al. (2021). Mitochondrial metabolic manipulation by SARS-CoV-2 in peripheral blood mononuclear cells of patients with COVID-19. Am. J. Phys. Cell Physiol. 320, C57-C65. doi: 10.1152/ajpcell.00426.2020

Alayash, A. I. (2004). Oxygen therapeutics: can we tame haemoglobin? Nat. Rev. Drug. Discov. 3, 152-159.

Alayash, A. I. (2014). Blood substitutes: why haven't we been more successful? Trends Biotechnol. 32, 177-185. doi: 10.1016/j.tibtech.2014.02.006

Andreux, P. A. (2013). Pharmacological approaches to restore mitochondrial function. Nat. Rev. Drug Discov. 12, 465-483. doi: 10.1038/nrd4023

Balcerek, B., Steinach, M., Lichti, J., Maggioni, M. A., Becker, P. N., Labes, R., et al. (2020). A broad diversity in oxygen affinity to haemoglobin. Sci. Rep. 10:16920. doi: 10.1038/s41598-020-73560-9

Bao, X., Zhang, J., Huang, G., Yan, J., Xu, C., Dou, Z., et al. (2021). The crosstalk between HIFs and mitochondrial dysfunctions in cancer development. Cell Death Dis. 12:215. doi: 10.1038/s41419-021-03505-1

Bickler, P. E., Feiner, J. R., Lipnick, M. S., Batchelder, P., MacLeod, D. B., and Severinghaus, J. W. (2017). Effects of acute, profound hypoxia on healthy humans: implications for safety of tests evaluating pulse oximetry or tissue oximetry performance. Anesth. Analg. 124, 146-153. doi: 10.1213/ANE.0000000000001421

Buehler, P. W., D'Agnillo, F., Hoffman, V., and Alayash, A. I. (2007). Effects of endogenous ascorbate on oxidation, oxygenation, and toxicokinetics of cell-free modified hemoglobin after exchange transfusion in rat and Guinea pig. J. Pharmacol. Exp. Ther. 323, 49-60. doi: 10.1124/jpet.107.126409

Burtscher, J., Cappellano, G., Omori, A., Koshiba, T., and Millet, G. P. (2020). Mitochondria: in the crossfire of SARS-CoV-2 and immunity. iScience 23:101631. doi: 10.1016/j.isci.2020.101631

Chacko, B. K., Kramer, P. A., Ravi, S., Benavides, G. A., Mitchell, T., Dranka, B. P., et al. (2014). The bioenergetic health index: a new concept in mitochondrial translational research. Clin. Sci. 127, 367-373. doi: 10.1042/CS20140101

Chowdhury, R., McDonough, M. A., Mecinović, J., Loenarz, C., Flashman, E., Hewitson, K. S., et al. (2009). Structural basis for binding of hypoxiainducible factor to the oxygen-sensing prolyl hydroxylases. Structure 17, 981-989. doi: 10.1016/j.str.2009.06.002

Daniel, Y., Beverley, J., Hunt, B. J., Retter, A., Henderson, K., Wilson, S., et al. (2020). Haemoglobin oxygen affinity in patients with severe COVID-19 infection. Br. J. Haematol. 190, e126-e127. doi: 10.1111/bjh.16888

DeMartino, A. W., Rose, J. J., Amdahl, M. B., Dent, M. R., Shah, F. A., Bain, W., et al. (2020). No evidence of hemoglobin damage by SARS-CoV-2 infection. Haematologica 105, 2769-2773. doi: 10.3324/haematol.2020.264267

Dhont, S., Derom, E., Braeckel, E. V., Depuydt, P., and Lambrecht, B. N. (2020). The pathophysiology of 'happy' hypoxemia in COVID-19. Respir. Res. 21:198. doi: 10.1186/s12931-020-01462-5

Dickerson, R. E., and Gies, I. (1993). Hemoglobin: Structure, Function, Evolution and Pathology. USA: Benjamin/Cumming.

Fukushi, M., Yoshinaka, Y., Matsuoka, Y., Hatakeyama, S., Ishizaka, Y., and Kirikae, T. (2012). Monitoring of S protein maturation in the endoplasmic reticulum by calnexin is important for the infectivity of severe acute respiratory syndrome coronavirus. J. Virol. 86, 11745-11753. doi: 10.1128/JVI. 01250-12

Geier, M. R., and Geier, D. A. (2020). Respiratory conditions in coronavirus disease 2019 (COVID-19): important considerations regarding novel treatment strategies to reduce mortality. Med. Hypotheses 140:109760. doi: 10.1016/j. mehy.2020.109760

Gheblawi, M., Wang, K., Viveiros, A., Nguyen, Q., Zhong, J.-C., Turner, A. J., et al. (2020). Angiotensin-converting enzyme 2: SARS-CoV-2 receptor and regulator of the renin-angiotensin system: celebrating the 20th anniversary of the discovery of ACE2. Circ. Res. 126, 1457-1475. doi: 10.1161/CIRCRESAHA.120.317015

\section{AUTHOR CONTRIBUTIONS}

The author confirms being the sole contributor of this work and has approved it for publication.

Gille, T., Sesé, L., Aubourg, E., Fabre, E. E., Cymbalista, F., Ratnam, K. C. et al. (2021). The affinity of hemoglobin for oxygen is not altered during COVID-19. Front. Physiol. 12:578708. doi: 10.3389/fphys.2021.578708

Gutiérrez, T., Qi, H., Yap, M. C., Tahbaz, N., Milburn, L. A., and Lucchinetti, E. (2020). The ER chaperone calnexin controls mitochondrial positioning and respiration. Sci. Signal. 13:eaax6660. doi: 10.1126/scisignal.aax6660

Jana, S., Heaven, M., and Alayash, A. I. (2021). Cell-free hemoglobin does not attenuate the effects of the SARS-CoV-2 S1 spike protein in vitro. Int J. Mol. Sci. 22:9041. doi: 10.3390/ijms22169041

Koch, A., Kähler, W., Klapa, S., Grams, B., and van Ooij, P. A. M. (2020). The conundrum of using hyperoxia in COVID-19 treatment strategies: may intermittent therapeutic hyperoxia play a helpful role in the expression of the surface receptors ACE2 and furin in lung tissue via triggering of HIF-1 $\alpha$ ? Intensive Care Med. Exp. 8:53. doi: 10.1186/s40635-020-00323-1

Kozlov, G., and Gehring, K. (2020). Calnexin cycle-structural features of the ER chaperone system. FEBS J. 287, 4322-4340. doi: 10.1111/febs. 15330

Kulow, V. A., and Fähling, M. (2021). How to increase cellular oxygen availability in COVID-19? Acta Physiol. 5:e13724. doi: 10.1111/apha.13724

Lee, P., Chandel, N. S., and Simon, M. C. (2020). Cellular adaptation to hypoxia through hypoxia inducible factors and beyond. Nat. Rev. Mol. Cell Biol. 21, 268-283. doi: 10.1038/s41580-020-0227-y

Liu, W., and Li, H. (2020). COVID-19: attacks the 1-beta chain of hemoglobin and captures the porphyrin to inhibit human heme metabolism. ChemRxiv [Preprint]. doi: 10.26434/chemrxiv.11938173.v8

Lupon, E., Lellouch, A. G., Franck, Z., Cetrulo, C. L., and Lantieri, L. A. (2021). Combating hypoxemia in COVID-19 patients with oxygen carrier, HEMO2Life (M101). Med. Hypotheses 146:110421. doi: 10.1016/j. mehy.2020.110421

Maltepe, E., and Saugstad, O. D. (2009). Oxygen in health and disease: regulation of oxygen homeostasis-clinical implications. Pediatr. Res. 65, 261-268. doi: 10.1203/PDR.0b013e31818fc83f

Manalo, D. J., Buehler, P. W., Baek, J. H., Butt, O., D’Agnillo, F., and Alayash, A. I. (2008). Acellular haemoglobin attenuates hypoxia-inducible factor- $1 \alpha$ (HIF- $1 \alpha)$ and its target genes in haemodiluted rats. Biochem. J. 414, 461-469. doi: 10.1042/BJ20080313

Martínez-Reyes, I., and Chandel, N. S. (2020). Mitochondrial TCA cycle metabolites control physiology and disease. Nat. Commun. 11:102. doi: 10.1038/s41467-019-13668-3

McLaren, A. T., Marsden, P. A., Mazer, C. D., Baker, A. J., Stewart, D. J., Tsui, A., et al. (2007). Increased expression of HIF-1alpha, nNOS, and VEGF in the cerebral cortex of anemic rats. Am. J. Phys. Regul. Integr. Comp. Phys. 292, R403-R414. doi: 10.1152/ajpregu.00403.2006

Nakhle, J., Rodriguez, A.-M., and Vignais, M.-L. (2020). Multifaceted roles of mitochondrial components and metabolites in metabolic diseases and cancer. Int. J. Mol. Sci. 21:4405. doi: 10.3390/ijms21124405

Pan, D., Mudalige, N. L., Oyefeso, O., and Barker, J. (2020). Blood transfusion strategies and ECMO during the COVID-19 pandemic. Lancet Respir. Med. 8:e40. doi: 10.1016/S2213-2600(20)30173-9

Peyssonnaux, C., Cejudo-Martin, P., Doedens, A., Zinkernagel, A. S., Johnson, R. S. and Nizet, V. (2007). Cutting edge: essential role of hypoxia inducible factor- $1 \alpha$ in development of lipopolysaccharide-induced sepsis. J. Immunol. 178, 7516-7519. doi: 10.4049/jimmunol.178.12.7516

Rahman, A., Tabassum, T., Araf, Y., Al Nahid, A., Ullah, M. A., and Hosen, M. J. (2021). Silent hypoxia in COVID-19: pathomechanism and possible management strategy. Mol. Biol. Rep. 48, 3863-3869. doi: 10.1007/ s11033-021-06358-1

Read, R. (2020). Flawed methods in "attacks the 1-beta chain of hemoglobin and captures the porphyrin to inhibit human heme metabolism". ChemRxiv [Preprint]. doi: 10.26434/chemrxiv.12120912.v2 
Selvaraj, P., Lien, C. Z. L., Liu, S., Stauft, C. B., Nunez, I. A., Hernandez, M., et al. (2021). SARS-CoV-2 infection induces protective immunity and limits transmission in Syrian hamsters. Life Sci. Alliance 4:e202000886. doi: 10.26508/ lsa. 202000886

Semenza, G. L. (2007). Life with oxygen. Science 318, 62-64. doi: 10.1126/science.1147949

Serebrovska, Z. O., Chong, E. Y., Serebrovska, T. V., Tumanovska, L. V., and Lei, X. I. (2020). Hypoxia, HIF-1 $\alpha$, and COVID-19: from pathogenic factors to potential therapeutic targets. Acta Pharmacol. Sin. 41, 1539-1546. doi: 10.1038/s41401-020-00554-8

Shenoy, S. (2020). Coronavirus (Covid-19) sepsis: revisiting mitochondrial dysfunction in pathogenesis, aging, inflammation, and mortality. Inflamm. Res. 69, 1077-1085. doi: 10.1007/s00011-020-01389-z

Thomas, T., Stefanoni, D., Dzieciatkowska, M., Issaian, A., Nemkov, T., and Hill, R. C. (2020). Evidence of structural protein damage and membrane lipid remodeling in red blood cells from COVID-19 patients. J. Proteome Res. 19, 4455-4469. doi: 10.1021/acs.jproteome.0c00606

Tobin, M. J., Laghi, F., and Jubran, A. (2020). Why COVID-19 silent hypoxemia is baffling to physicians? Am. J. Respir. Crit. Care Med. 202, 356-360. doi: 10.1164/rccm.202006-2157CP

Vassilaki, N., and Frakolaki, E. (2017). Virus-host interactions under hypoxia. Microbes Infect. 19, 193-203. doi: 10.1016/j.micinf.2016.10.004

Vogel, D. J., Formenti, F., Retter, A. J., Vasques, F., and Camporota, L. (2020). A left shift in the oxyhaemoglobin dissociation curve in patients with severe coronavirus disease 2019 (COVID-19). Br. J. Haematol. 191, 390-393. doi: 10.1111/bjh.17128
Weiskopf, R. B., Glassberg, E., Guinn, M. H. A., James, M. F. M., and Ness, P. M. (2020). The need for an artificial oxygen carrier for disasters and pandemics, including COVID-19. Transfusion 60, 3039-3045. doi: 10.1111/trf. 16122

Woyke, S., Rauch, S., Ströhle, M., and Gatterer, H. (2021). Modulation of $\mathrm{Hb}_{2} \mathrm{O}_{2}$ affinity to improve hypoxemia in COVID-19 patients. Clin. Nutr. 40, 38-39. doi: 10.1016/j.clnu.2020.04.036

Conflict of Interest: The author declares that the research was conducted in the absence of any commercial or financial relationships that could be construed as a potential conflict of interest.

Publisher's Note: All claims expressed in this article are solely those of the authors and do not necessarily represent those of their affiliated organizations, or those of the publisher, the editors and the reviewers. Any product that may be evaluated in this article, or claim that may be made by its manufacturer, is not guaranteed or endorsed by the publisher.

Copyright (c) 2021 Alayash. This is an open-access article distributed under the terms of the Creative Commons Attribution License (CC BY). The use, distribution or reproduction in other forums is permitted, provided the original author(s) and the copyright owner(s) are credited and that the original publication in this journal is cited, in accordance with accepted academic practice. No use, distribution or reproduction is permitted which does not comply with these terms. 